\title{
Application of C-reactive Protein/Albumin Ratio (CAR) in Kawasaki Disease
}

\section{Zhenquan Wang}

Second Affiliated Hospital \& Yuying Children's Hospital of Wenzhou Medical University

\section{Yiping Shao}

Second Affiliated Hospital \& Yuying Children's Hospital of Wenzhou Medical University

\section{Xing Rong}

Second Affiliated Hospital \& Yuying Children's Hospital of Wenzhou Medical University

Huixian Qiu

Second Affiliated Hospital \& Yuying Children's Hospital of Wenzhou Medical University

Jinxing Wang

Second Affiliated Hospital \& Yuying Children's Hospital of Wenzhou Medical University

\section{Xuting Zhang}

Second Affiliated Hospital \& Yuying Children's Hospital of Wenzhou Medical University

\section{Biyao Hang}

Second Affiliated Hospital \& Yuying Children's Hospital of Wenzhou Medical University

\section{Zhipeng Xu}

Second Affiliated Hospital \& Yuying Children's Hospital of Wenzhou Medical University

Rongozhou Wu

Second Affiliated Hospital \& Yuying Children's Hospital of Wenzhou Medical University

Maoping Chu ( $\nabla$ chmping@hotmail.com)

Second Affiliated Hospital \& Yuying Children's Hospital of Wenzhou Medical University

\section{Research Article}

Keywords: Kawasaki disease, C-reactive protein, albumin ratio, CAR, Coronary artery lesions, IVIG resistance

Posted Date: March 16th, 2021

DOI: https://doi.org/10.21203/rs.3.rs-284753/v1

License: (c) (i) This work is licensed under a Creative Commons Attribution 4.0 International License. Read Full License 


\title{
Application of C-reactive Protein/Albumin Ratio (CAR) in Kawasaki Disease
}

Zhenquan Wang", Yiping Shao", Xing Rong, Huixian Qiu, Jinxing Wang, Xuting Zhang, Biyao Hang, Zhipeng Xu, Rongozhou Wu, Maoping Chu*

Children's Heart Center, The Second Affiliated Hospital and Yuying Children's Hospital, Institute of

Cardiovascular Development and Translational Medicine, Wenzhou Medical University, Wenzhou 325027,

Zhejiang, China

Zhenquan Wang ${ }^{\#}$ and Yiping Shao contributed equally to this work

Correspondence should be addressed to Maoping Chu; chmping@hotmail.com

\begin{abstract}
Objective: To investigate the association between the C-reactive protein/albumin ratio (CAR) and coronary artery lesions (CAL), intravenous immunoglobulin (IVIG) resistance in children with Kawasaki disease (KD).
\end{abstract}

Methods: We retrospectively studied 753 children with $\mathrm{KD}$, categorizing them into the CAL $\operatorname{group}(n=238)$ and the No-CAL group $(n=515)$, the IVIG-resistance group $(n=61)$ and the No-IVIGresistance $(n=653)$ group. The differences in laboratory data, clinical manifestations, the relationship between CAR and CAL as well as IVIG resistance were compared between the two cohort groups.

Results: Compared with No-CAL group, KD with CAL had a higher CAR $(2.12$ vs 1.69 , p <0.001). And CAR was significantly higher in KD children with IVIG resistance $(2.42$ vs $1.85, \mathrm{p}<0.001)$. Multivariable logistic regression analysis demonstrated that higher CAR was a risk factors of CAL 
$(\mathrm{OR}=1.198, \mathrm{p}<0.001)$ and $\mathrm{IVIG}$ resistance $(\mathrm{OR}=1.297, \mathrm{p}<0.001)$, respectively. CAL and IVIG resistance interact with each other. ROC curve analysis performed for the prediction of CAL, the best cut-off point for CAR was $1.80(\mathrm{AUC}=0.602$, sensitivity $64.7 \%$, specificity $54.8 \%$ ). When predicting IVIG resistance according to the ROC curve analysis, the optimal cutoff point for CAR was $2.20(\mathrm{AUC}=0.621$, sensitivity $59.0 \%$, specificity $61.1 \%)$.

Conclusions: CAR is a valid indicator in KD children. Higher CAR may be helpful in predicting CAL and IVIG resistance in KD.

Keywords: Kawasaki disease, C-reactive protein, albumin ratio, CAR, Coronary artery lesions, IVIG resistance

\section{Introduction}

Kawasaki disease (KD) is an extensive inflammation of small and medium blood vessels throughout the body. The major serious complication of KD is coronary artery lesions (CAL), which is also the most concerned and focused part to the majority of researchers. As an acute, self-limited febrile illness, KD predominantly affects children $<5$ years of age ${ }^{[1]}$. Timely intravenous administration of high-dose immunoglobulin (IVIG) can reduce the risk of heart disease complications, but CAL still occurs in $5 \%$ of $\mathrm{KD}$ children, making it a major cause of childhood acquired heart disease ${ }^{[2]}$. In addition, about $10-20 \%$ of KD patients expressed IVIG resistance, despite the initial IVIG therapy, which increases the risk of cardiac complications ${ }^{[3]}$. In recent years, the incidence rate reported in Japan and China has been increasing year by year ${ }^{[4-7]}$. How to identify early Cal and IVIG resistance in $\mathrm{KD}$ is very important for clinical work.

Inflammation plays an important role in KD. C-reactive protein (CRP) and albumin, commonly used 
as inflammatory markers, are known as positive and negative acute reaction products, respectively, and are considered to be associated with coronary artery lesions and adverse cardiovascular events. The newly introduced parameter $\mathrm{CRP} /$ albumin ratio (CAR) is considered to be more accurate than CRP or albumin alone in responding to inflammatory states ${ }^{[8]}$. It reflects the balance between CRP and albumin levels and has been used to assess coronary artery lesions and its severity, monitor rheumatoid arthritis disease activity and the prognosis of cancer patients ${ }^{[9-11]}$. CRP and/or albumin alone have been investigated in $\mathrm{KD}$, but it is not clear whether CAR is associated with CAL and IVIG resistance. The purpose of this study was to investigate the relationship between CAR and coronary artery lesions as well as IVIG resistance among KD children.

\section{Materials and Methods}

\subsection{Subject}

We reviewed the data of children with KD at the Second Affiliated Hospital of Wenzhou Medical University from 1 January 2018 to 31 December 2019 .Overall, 818 were collected, 46 excluded due to the following reasons: 3 children received the initial treatment before hospitalization, 19 patients were recurrent cases, 5 children were in convalescence of KD and the data were incomplete in 38 patients. Finally, 772 children were included in the study. We classified all KD children into the non-coronary artery lesions group (No-CAL group, $n=515$ ) and the coronary artery lesions group (CAL group, $\mathrm{n}=238$ ) according to their coronary artery status. Then, according to the resistance of IVIG, they were divided into the IVIG non-resistance $\operatorname{group}(\mathrm{n}=653)$ and the IVIG resistance $\operatorname{group}(\mathrm{n}=61)$.

All children were diagnosed with reference to the Scientific Statement for Health Professionals 
From the American Heart Association ${ }^{[1]}$. Children who met the 5 or $6 / 6$ diagnostic criteria (fever $>5$ days; extremity changes; rash conjunctivitis; oral changes; cervical lymphadenopathy) were defined as $\mathrm{KD}$, and those with less than 5 diagnostic criteria were defined as atypical KD. All the children received echocardiography before treatment, 2 weeks after onset, and 3-4 weeks after onset.

\subsection{Data Collection and Laboratory Tests}

We collected the following information: age (month), sex, time and method of IVIG treatment, and IVIG response. Before the IVIG treatment, we collected laboratory data including white blood cell count, hemoglobin level, neutrophil count, platelet levels, lymphocyte count, eosinophil count and levels of ALT, AST, erythrocyte sedimentation rate, fibrinogen, D-dimer, brain natriuretic peptide and c-reactive protein (CRP) and serum albumin. And calculate the CAR value (CRP/albumin). At least 3 times of Echocardiography y should be performed.

\subsection{Resistance to IVIG and CAL}

Patients develop recrudescent or persistent fever at least 36 hours after the end of first IVIG infusion (body temperature $>37.3^{\circ} \mathrm{C}$ ) were defined as IVIG resistance $\mathrm{e}^{[1]}$.

Echocardiography was used to assess CAL. CAL are defined according to the Z-Score recommended in the guidelines ${ }^{[1]}$. The Z-Score is classified as follows. (1) No involvement: Always, $<2$; (2) Dilation only: 2 to $<2.5$; or if initially $<2$, a decrease in $\mathrm{Z}$ score during follow-up $\geq 1$; (3) Small aneurysm: $\geq 2.5$ to $<5$; (4) Medium aneurysm: $\geq 5$ to $<10$, and absolute dimension $<8 \mathrm{~mm}$; (5) Large or giant aneurysm: $\geq 10$, or absolute dimension $\geq 8 \mathrm{~mm}$. Any one of the three 
echocardiographic results suggested that coronary artery lesions, including aneurysmal dilatation, were included in the CAL group.

\subsection{Statistical Analysis}

SPSS version 22.0 was used for statistical analysis. count data were expressed as the

Mean \pm standard deviation or median and interquartile range, and measurement data were expressed as numbers and percentages. Categorical variables were compared using the Chi-square test. The receiver operating characteristic curve was used to examine the predictive value of CAR in patients with CAL and IVIG resistance. The area under the curve was calculated. In the ROC curve, the sensitivity and specificity, for the prediction of CAL and IVIG resistance were calculated by combining the optimal cut-off values for each index using the Youden index. Logistic regression analysis was used to identify predictors of KD patient with CAL and IVIG resistance. The results were considered statistically significant when $\mathrm{p}$ values were $<0.05$.

\section{Result}

\subsection{Demographic Characteristics and Clinical Data}

As shown in table 1, the proportion of male children in CAL group was slightly higher than that in No-CAL group $(P=0.049)$, and the age was slightly younger (17.4months vs 24.4 months; $P<0.001)$. The proportion of main clinical symptoms such as oral changes and lymph in CAL group was lower than that in No-CAL group $(85.5 \%$ vs $93.8 \%, p<0.001 ; 28.2 \%$ vs $39.9 \%, \quad P=0.002$, respectively), and there was no statistical difference in other clinical manifestations. In addition, typical KD was dominant in CAL group, but the proportion was lower than that in No-CAL group $(57.1 \%$ vs $64.9 \% ; P=0.042)$. The treatment duration of IVIG in both 
groups was 6 days, but there was no statistically significant difference.The proportion of IVIG resistance in CAL group was higher than that in No-CAL group $(15.7 \%$ vs $5.20 \% ; P<0.001)$.

\subsection{CAL group Laboratory Tests}

As shown in table 2, the levels of $\mathrm{Hb}$ and Hct were slightly lower $(111 \pm 9.97 \mathrm{~g} / \mathrm{l} \mathrm{vs} 115 \pm 10.2 \mathrm{~g} / \mathrm{l}$, $P<0.001 ; 0.335 \pm 0.030 \%$ vs $0.347 \pm 0.030 \%, P<0.001$, respectively) in CAL group. In the liver function test, compare with No-CAL group, the level of ALT and $\gamma$-GT were higher in CAL group $(36.0 \mu / L$ vs $28.0 \mu / L, P=0.017 ; 52.0 \mu / L$ vs $26.0 \mu / L, P=0.003$, respectively). Other indexes were no significant difference between the two groups. In the indexes of coagulation function test, the level of PT and D-dimers were significantly higher in CAL group than that in No-CAL group $(14.2 \pm 1.11 \mathrm{~s}$ vs $13.8 \pm 1.08 \mathrm{~s}, P<0.001 ; 1.47 \mu \mathrm{g} / \mathrm{ml}$ vs $1.31 \mu \mathrm{g} / \mathrm{ml}, P=0.040$, respectively).The levels of NT-pro BNP were significantly higher in CAL group than in No-CAL group $(836 \mathrm{pg} / \mathrm{ml} \mathrm{vs} 509 \mathrm{pg} / \mathrm{ml}, P<0.001)$.

In addition, the levels of CRP in CAL group was higher than that in No-CAL group $(84.0 \mathrm{mg} / \mathrm{L} v \mathrm{~s}$ $66.8 \mathrm{mg} / \mathrm{L}, P<0.001)$, but the level of albumin was lower(39.0g/L vs $40.8 \mathrm{~g} / \mathrm{L}, P<0.001)$. And the level of CAR was significantly higher in CAL group than in No-CAL group $(2.12$ vs $1.69, P<0.001)$.

\subsection{IVIG Resistance group Laboratory Tests.}

As shown in table 2, there was no statistical difference in blood routine indexes. In the liver function test, the level of ALT and $\gamma$-GT were significantly higher in IVIG resistance group than in the No-IVIG resistance group $(56.0 \mu / L$ vs $31.0 \mu / L, P=0.017 ; 67.5 \mu / L$ s $32.0 \mu / L, P<0.001)$. Among the coagulation function indexes, only the level of D-dimers was significantly higher in IVIG resistance group than that in No-IVIG resistance group $(2.10 \mu \mathrm{g} / \mathrm{ml} v \mathrm{~s} 1.33 \mu \mathrm{g} / \mathrm{ml}, \quad P=0.04)$, while there was no difference in other indexes. And the level of NT-pro BNP was significantly higher in IVIG resistance group than in No-IVIG resistance group $(1320 \mathrm{pg} / \mathrm{ml} \mathrm{vs} 609 \mathrm{pg} / \mathrm{ml}, P<0.001)$. 
In addition, compared with No-IVIG resistance group, the levels of CRP was significantly higher $(92.6 \mathrm{mg} / \mathrm{L}$ vs $74.0 \mathrm{mg} / \mathrm{L}, P<0.001)$ and the level of albumin was lower( $38.0 \mathrm{~g} / \mathrm{Lvs} 40.5 \mathrm{~g} / \mathrm{L}, P<0.001)$ in IVIG resistance group. And the level of CAR was significantly higher in IVIG resistance $\operatorname{group}(2.42$ vs $1.86, P<0.001)$.

\subsection{CAR, Coronary Artery Outcomes and IVIG Resistance}

\subsubsection{CAR and Coronary Artery Lesions}

Logistic regression analysis was performed for the correlation between CAR and CAL (Table 3). Elevated CAR and IVIG resistance $(+)$ were independent risk factors for CAL. When we adjusted for possible confounding factors that might be associated with CAL, such as age, sex and IVIG treat time, high level of CAR $(\mathrm{OR}=1.198,95 \% \mathrm{CI}=1.086-1.322, P<0.001)$ and IVIG resistance $(+)$ $(\mathrm{OR}=3.439,95 \% \mathrm{CI}=1.741-5.238, P<0.001)$ remained a significant risk factor for CAL. ROC curves were constructed to determine the discriminatory ability of CAR to predict the occurrence of CAL (Fig 1, Table4), CAR(AUC $=0.602)$, with a sensitivity of $64.7 \%$ and a specificity of $54.8 \%$, showed a better performance than CRP $(\mathrm{AUC}=0.590)$. The cut-off value of CAR for CAL in KD children was 1.80 .

\subsubsection{CAR and IVIG Resistance}

Logistic regression analysis was performed for the correlation between CAR and VIG Resistance (Table 5). The results showed that CAL (+) before IVIG and high level of CAR were independent risk factors for IVIG resistance. And after we adjusted for possible confounding factors, such as as age, sex and IVIG treat time, increased CAR $(\mathrm{OR}=1.266,95 \% \mathrm{CI}=1.094-1.464, \mathrm{P}=0.002)$ and $\mathrm{CAL}$ 
$(+)$ before IVIG $(\mathrm{OR}=3.096,95 \% \mathrm{CI}=1.784-5.373, \mathrm{P}<0.001)$ remained a significant risk factor for IVIG resistance.

ROC curves were constructed to determine the discriminatory ability of CAR to predict the occurrence of IVIG resistance (Fig 2, Table 6), CAR(AUC=0.621), with a sensitivity of $59.0 \%$ and a specificity of $61.1 \%$, showed a better performance than CRP (AUC=0.603). The cut-off value of CAR for CAL in KD children was 2.20.

\section{Discussion}

KD has been around for decades. Unfortunately, the pathogenesis of KD is still unclear. However, $\mathrm{KD}$ is now known to be characterized by systemic inflammation in all the medium-sized arteries and in multiple organs and tissues during the acute febrile phase ${ }^{[12]}$. In recent years, the incidence of KD in Japan, South Korea and other regions has shown an increasing trend, and China is no exception ${ }^{[4,5]}$. Even with IVIG treatment following strict guidelines, approximately $4.9 \%$ of patients with KD develop coronary artery abnormalities ${ }^{[13]}$. According to reports from Beijing, about $12.8 \%$ of KD patients have IVIG resistance ${ }^{[14]}$. And IVIG resistors are prone to develop CAL ${ }^{[15]}$. Thus, early identification of IVIG resistance and risk factors for coronary artery lesions is crucial for pediatricians to actively treat KD and improve prognosis.

$\mathrm{CRP}$, an acute-phase reactant protein, is a sensitive indicator of inflammation, and its change is often associated with the degree of inflammation ${ }^{[16]}$. It can be frequently used for the diagnosis, treatment follow-up and prognosis of inflammatory diseases ${ }^{[17]}$. In the acute phase of inflammatory diseases, the phenomenon of hypoalbuminemia may be caused by the following reasons: the decreased ability of the liver to produce albumin ${ }^{[18,19]}$. Alternatively, inflammatory cells release 
cytokines that increase microvascular permeability and serum albumin extravasation from the blood vessels $^{[20]}$. Therefore, albumin is also considered to be closely related to inflammation severity and disease prognosis ${ }^{[21]}$. Furthermore, related findings ${ }^{[22]}$ found that the hypoalbuminemia reflects outcome which CRP and other inflammatory markers can not. Although CRP and albumin are good indicators of inflammation, while previous studies have shown that the CAR is more predictive than CRP or albumin alone ${ }^{[23-25]}$. The CAR is a novel inflammation-based prognostic score, which is correlated to the inflammation severity and mortality ${ }^{[24,26]}$, and is often used to predict the prognosis of disease such as cancer, sepsis, acute pancreatitis ${ }^{[24,27,28]}$ etc. Higher CAR levels are associated with higher inflammatory status and poorer prognosis ${ }^{[24,29]}$.

In our study, we found that compared with the No-CAL group, KD children in the CAL group had higher CAR, and CAR may help to predict the occurrence of CAL. Through regression analysis, we found that high CAR, high CRP and low albumin were risk factors for CAL in children with KD, even after controlling for age, sex and IVIG treat time. High CRP levels and low albuminemia are reported to be risk factors for CAL development ${ }^{[30]}$. Our results are consistent with that. CRP stimulates leucocyte-endothelial interaction by regulating endothelium-derived molecules and chemokines, and impairs the number and function of endothelial progenitor cells by promoting apoptosis and attenuating their migration and adherence capacities ${ }^{[31]}$. The level of CRP is not only a manifestation of vascular inflammation, but also a good predictor of future cardiovascular events $^{[32,33]}$. Albumin has the ability of antioxidation and free radical-scavenging, capacity to prevent apoptosis, and affinity for binding with lipids, drugs, toxic substances and other ligands ${ }^{[22]}$. So, the albumin may have potential clinical biological protective effects ${ }^{[22]}$. CAR combines information provided by CRP and albumin to predict coronary artery disease. We found through 
ROC curve analysis that CRP had a sensitivity of $63.0 \%$ and specificity of $54.2 \%$ for predicting CAL at cutoff value of $71.0 \mathrm{mg} / \mathrm{L}$ but CAR had a sensitivity of $64.7 \%$ and specificity of $54.8 \%$ for predicting CAL $>1.80$ cutoff value which shows better accuracy of CAR in predicting CAL than CRP. Therefore, the higher the CAR, the higher ranking risk of CAL.

Our study also found that CAR was associated with IVIG resistance and was a predictor of IVIG resistance in KD children. IVIG is the most effective first-line treatment during the acute phase of KD. The therapeutic efficacy of IVIG might be achieved through reduction of systemic immune response and levels of related cytokines ${ }^{[34]}$. However, approximately $10 \%$ to $20 \%$ of patients with KD have persistent or recurrent fever after primary therapy with IVIG plus aspirin ${ }^{[1]}$. So far, there is no consensus regarding the risk factors of IVIG resistance. Based on the reported literature ${ }^{[15,35]}$, laboratory levels such as increased CRP and decreased albumin, reduced Hb level, elevated WBC count, increased level of LDH, ESR and PLT could serve as independent risk factors for predicting IVIG resistance. Compared to IVIG-responder, IVIG resistance showed a more intense inflammatory response. Increased microvascular permeability is an important event during the initial process of KD vasculitis ${ }^{[36]}$. Hypoalbuminemia caused by vascular leakage is a key feature of pathophysiology of KD. AS Terai et al ${ }^{[37]}$ revealed that hypoproteinemia was much serious in the IVIG-resistance group than in the IVIG-responsive group. Plasma albumin is a constitutive protein. And the albumin levels are valuable indicators of recovery or deterioration of health ${ }^{[38]}$. IVIG resistant children expressed more severe vascular leakage and liver damage. Hypoalbuminemia, is the result and manifestation of inflammation, which indicate the degree of vasculitis, and is associated with IVIG resistance. CAR combines the acute course of inflammation with the chronic outcome nutritional status to reflect the degree of inflammation and the prognosis of the disease. 
Thus, CAR is considered to be a good indicator of the state of inflammation. We further examined the association between CRP, CAR, albumin and IVIG resistance. Our data demonstrated that the optimal cut-off point of the CAR for IVIG resistance was 2.20 , with a sensitivity of $59.0 \%$ and a specificity of $61.1 \%$, and the AUC of the CAR was 0.621 , which was higher than that of CRP (AUC 0.603). Therefore, we believed that CAR was associated with IVIG resistance and may be used as a potential predictor of IVIG resistance.

In the study cohort, the CAL group was significantly younger than the No-CAL group, and mainly male, which means CAL mainly affected younger and male patients. In our study, we found that nearly $8.28 \%$ of the patients showed immunoglobulin resistance, slightly lower than the previously reported drug resistance rate $(10 \%-20 \%)^{[14,30]}$. This may be related to the time of our first dose of IVIG treatment. There were $74.4 \%$ of the children with KD were treated with IVIG on the 5 th- 7 th day of the disease. Moreover, we found that the IVIG resistance rate $(11.0 \%$ VS $7.30 \%)$ was significantly higher in CAL group than in the No-CAL group, and the incidence of coronary artery lesions in the IVIG resistance was $47.3 \%$, similar to the previously reported studies $(47.5 \%)^{[3]}$. After all, patients with IVIG resistance are at high risk of developing coronary artery lesions ${ }^{[39]}$.

Our study also found that, in addition to CAR can being a predictor for KD's occurrence of CAL and IVIG resistance, CAL and IVIG resistance also interact with each other. (1) The occurrence of CAL before treatment may contribute to IVIG resistance. At present, the pathogenesis of Kawasaki disease complicated with CAL is not fully understood. Some scholars believed that oxidative stress, immune activation, and the coronary arteries infiltration of immune system cells associated with secretion of proinflammatory cytokines, elastases, and matrix metalloproteinases in contribute to the occurrence of $\mathrm{CAL}^{[40-43]}$. The occurrence of CAL before IVIG treatment is a signal of strong 
vascular inflammation, which means that vascular leakage is serious, resulting in increased albumin leakage, and thereby increasing the risk of IVIG resistance ${ }^{[37]}$. A study from Children's Hospital Colorado $^{[44]}$ suggested that Children with $\mathrm{KD}$ who had CAL at the time of diagnosis were more likely to have IVIG resistance, which is consistent with our findings. (2) IVIG resistance is associated with an increased risk for CAL in children with KD. Systemic inflammatory response is severe in KD children with IVIG resistance, the increase of cytokines damages vascular endothelium, increases vascular endothelial permeability, causes perivascular edema, and then aggravates vascular wall injury ${ }^{[45]}$. Albumin not only indicates nutritional status but also a useful factor for predicting patient prognosis ${ }^{[21]}$. Thus, IVIG-resistant patients are at higher risk of developing CAL than IVIG-responsive patients ${ }^{[34,46]}$. In summary, attention should be paid to the KD children with CAL before treatment or IVIG resistance after the first dose of treatment. Further research is needed to determine whether pediatricians may consider combining IVIG and aspirin with second-line treatments such as hormones and immunosuppressants to reduce the incidence of IVIG resistance and CAL.

Our study has several limitations. Firstly, our study is a single-center study with a time span of only 2 years. A larger study is needed to confirm our findings. Secondly, whether the predictive role of CAR in KD coronary damage and IVIG resistance is applicable in other populations needs to be verified by larger studies. Finally, due to the different time of visit and treatment for KD children, blood sample collection and determination time were different before treatment, we could not evaluate the changes in CRP, albumin, and CAR over time. This may be the reason why CAR specificity and sensitivity are not high enough. Further studies that investigate the changes in CAR are needed in the future. 


\section{Conclusion}

Our study found that CAR can predict the occurrence of CAL and IVIG resistance in KD to some extent. Therefore, for hospitalized children diagnosed with KD, clinicians should pay attention to CAR value, especially for children with acute stage and high CAR value, and adopt more active treatment measures and cardiac ultrasound follow-up observation.

Table 1: Demographic Characteristics and Clinical Data

Table 2: Laboratory Tests

Table 3: Multiple regression analysis of Coronary Artery Lesions

Table 4: ROC curves of CAR and Coronary Artery Lesions

Figure 1: ROC curves of CAR and Coronary Artery Lesions

Table 5: Multiple regression analysis of IVIG Resistance

Table 6: ROC curve distribution of CAR and IVIG Resistance

Figure 2: ROC curves of CAR and IVIG Resistance 


\section{Declarations:}

\section{Ethics approval and consent to participate}

This study was approved by the ethical Board of Wenzhou Medical University, Zhejiang, China. Informed consent was signed by the parents of all patients. And all the experiment protocol for involving humans data was in accordance with the tenets of the Declaration of Helsinki.

\section{Consent for publication}

Not applicable.

\section{Data Availability}

The datasets generated and analysed during the current study are not publicly available due legal reasons. To deposit data in an open depository or send data to a journal where other people (you do not know whom) may access data MAY result in harm.Hence, researchers are not allowed to deposit the data elsewhere. But the data can be made available from the corresponding author on reasonable request.

\section{Conflicts of Interests}

The authors declare that they have no conflicts of interest.

\section{Funding}

The work was supported by the Natural Science Foundation of China (No. 81970435), and Zhejiang Provincial Science and Technology Project of Traditional Chinese Medicine (No. 2018ZZ019). 


\section{Authors' Contributions}

ZQW and YPS conceptualized and designed the study, collected the data, drafted the initial manuscript. JXW and XTZ carried out the statistics. BYH, ZPX made substantial contributions to the design of the paper, and its interpretation. XR and HXQ reviewed and revised the manuscript for important intellectual content. RZW and MPC reviewed and revised the manuscript. All authors read and contributed to the present manuscript. All authors read and approved the final manuscript.

\section{Acknowledgements}

Not applicable. 


\section{References}

[1] Bw Mccrindle, Ah Rowley, Jw Newburger et al. Diagnosis, Treatment, and Long-Term Management of Kawasaki Disease: A Scientific Statement for Health Professionals From the American Heart Association[J]. Circulation, 2017, 135(17): e927-e999.10.1161/cir.0000000000000484

[2] Ls Chang, Yj Lin, Jh Yan et al. Neutrophil-to-lymphocyte ratio and scoring system for predicting coronary artery lesions of Kawasaki disease[J]. BMC pediatrics, 2020, 20(1): 398.10.1186/s12887-020-02285-5

[3] By Kim, D Kim, Yh Kim et al. Non-Responders to Intravenous Immunoglobulin and Coronary Artery Dilatation in Kawasaki Disease: Predictive Parameters in Korean Children[J]. Korean circulation journal, 2016, 46(4): 542-549.10.4070/kcj.2016.46.4.542

[4] X Zhang, Y Liang, W Feng et al. Epidemiologic survey of Kawasaki disease in Inner Mongolia, China, between 2001 and 2013[J]. Experimental and therapeutic medicine, 2016, 12(2): 12201224.10.3892/etm.2016.3393

[5] Jh Piao, Lh Jin, J Lv et al. Epidemiological investigation of Kawasaki disease in Jilin province of China from 2000 to 2008[J]. Cardiology in the young, 2010, 20(4): 426-432.10.1017/s1047951110000375

[6] N. Makino, Y. Nakamura, M. Yashiro et al. Nationwide epidemiologic survey of Kawasaki disease in Japan, 2015-2016[J]. Pediatr Int, 2019, 61(4): 397-403.10.1111/ped.13809

[7] N. Makino, Y. Nakamura, M. Yashiro et al. Epidemiological observations of Kawasaki disease in Japan, 2013-2014[J]. Pediatr Int, 2018, 60(6): 581-587.10.1111/ped.13544

[8] M Çağdaş, I Rencüzoğullari, S Karakoyun et al. Assessment of Relationship Between C-Reactive Protein to Albumin Ratio and Coronary Artery Disease Severity in Patients With Acute Coronary Syndrome[J]. Angiology, 2019, 70(4): 361-368.10.1177/0003319717743325

[9] Z Tanriverdi, F Gungoren, Mb Tascanov et al. Comparing the Diagnostic Value of the C-Reactive Protein to Albumin Ratio With Other Inflammatory Markers in Patients With Stable Angina Pectoris[J]. Angiology, 2020, 71(4): 360-365.10.1177/0003319719897490

[10] W. M. Yang, W. H. Zhang, H. Q. Ying et al. Two new inflammatory markers associated with disease activity score-28 in patients with rheumatoid arthritis: Albumin to fibrinogen ratio and C-reactive protein to albumin ratio[J]. Int Immunopharmacol, 2018, 62: 293-298.10.1016/j.intimp.2018.07.007

[11] M. H. Kim, J. Y. Ahn, J. E. Song et al. The C-Reactive Protein/Albumin Ratio as an Independent Predictor of Mortality in Patients with Severe Sepsis or Septic Shock Treated with Early Goal-Directed Therapy[J]. PLoS One, 2015, 10(7): e0132109.10.1371/journal.pone.0132109

[12] S. Amano, F. Hazama, H. Kubagawa et al. General pathology of Kawasaki disease. On the morphological alterations corresponding to the clinical manifestations[J]. Acta Pathol Jpn, 1980, 30(5): 681-694.

[13] N. Michihata, H. Matsui, K. Fushimi et al. Guideline-Concordant Treatment of Kawasaki Disease With Immunoglobulin and Aspirin and the Incidence of Coronary Artery Aneurysm[J]. Clin Pediatr (Phila), 2015, 54(11): 1076-1080.10.1177/0009922814566932

[14] ZD Du, YL Zhang D Zhao et al. Treatment of intravenous immunoglobulin non-response patients and analysis of risk factors[J]. Chinese Journal of practical Pediatrics, 2006, (10): 738-741.

[15] M. Fukunishi, M. Kikkawa, K. Hamana et al. Prediction of non-responsiveness to intravenous high-dose gamma-globulin therapy in patients with Kawasaki disease at onset[J]. J Pediatr, 2000, 137(2): 172$176.10 .1067 / \mathrm{mpd} .2000 .104815$ 
[16] C. Rey, M. Los Arcos, A. Concha et al. Procalcitonin and C-reactive protein as markers of systemic inflammatory response syndrome severity in critically ill children[J]. Intensive Care Med, 2007, 33(3): 477-484.10.1007/s00134-006-0509-7

[17] C. Lelubre, S. Anselin, K. Zouaoui Boudjeltia et al. Interpretation of C-reactive protein concentrations in critically ill patients[J]. Biomed Res Int, 2013, 2013: 124021.10.1155/2013/124021

[18] P. W. Kowalski-Saunders, P. J. Winwood, M. J. Arthur et al. Reversible inhibition of albumin production by rat hepatocytes maintained on a laminin-rich gel (Engelbreth-Holm-Swarm) in response to secretory products of Kupffer cells and cytokines[J]. Hepatology, 1992, 16(3): 733-741.10.1002/hep.1840160320

[19] M. D. Barber, J. A. Ross K. C. Fearon. Changes in nutritional, functional, and inflammatory markers in advanced pancreatic cancer[J]. Nutr Cancer, 1999, 35(2): 106-110.10.1207/s15327914nc352_2

[20] G. Fanali, A. Di Masi, V. Trezza et al. Human serum albumin: from bench to bedside[J]. Mol Aspects Med, 2012, 33(3): 209-290.10.1016/j.mam.2011.12.002

[21] D. Gupta C. G. Lis. Pretreatment serum albumin as a predictor of cancer survival: a systematic review of the epidemiological literature[J]. Nutr J, 2010, 9: 69.10.1186/1475-2891-9-69

[22] J. L. Vincent, M. J. Dubois, R. J. Navickis et al. Hypoalbuminemia in acute illness: is there a rationale for intervention? A meta-analysis of cohort studies and controlled trials[J]. Ann Surg, 2003, 237(3): 319334.10.1097/01.Sla.0000055547.93484.87

[23] J. Oh, S. H. Kim, K. N. Park et al. High-sensitivity C-reactive protein/albumin ratio as a predictor of inhospital mortality in older adults admitted to the emergency department[J]. Clin Exp Emerg Med, 2017, 4(1): 19-24.10.15441/ceem.16.158

[24] O. T. Ranzani, F. G. Zampieri, D. N. Forte et al. C-reactive protein/albumin ratio predicts 90-day mortality of septic patients[J]. PLoS One, 2013, 8(3): e59321.10.1371/journal.pone.0059321

[25] J. E. Park, K. S. Chung, J. H. Song et al. The C-Reactive Protein/Albumin Ratio as a Predictor of Mortality in Critically Ill Patients[J]. J Clin Med, 2018, 7(10): 10.3390/jcm7100333

[26] T. Zhou, J. Zhan, S. Hong et al. Ratio of C-Reactive Protein/Albumin is An Inflammatory Prognostic Score for Predicting Overall Survival of Patients with Small-cell Lung Cancer[J]. Sci Rep, 2015, 5: 10481.10.1038/srep10481

[27] M. Kaplan, I. Ates, M. Y. Akpinar et al. Predictive value of C-reactive protein/albumin ratio in acute pancreatitis[J]. Hepatobiliary Pancreat Dis Int, 2017, 16(4): 424-430.10.1016/s1499-3872(17)60007-9

[28] M. Ishizuka, H. Nagata, K. Takagi et al. Clinical Significance of the C-Reactive Protein to Albumin Ratio for Survival After Surgery for Colorectal Cancer[J]. Ann Surg Oncol, 2016, 23(3): 900907.10.1245/s10434-015-4948-7

[29] Z. Liu, K. Jin, M. Guo et al. Prognostic Value of the CRP/Alb Ratio, a Novel Inflammation-Based Score in Pancreatic Cancer[J]. Ann Surg Oncol, 2017, 24(2): 561-568.10.1245/s10434-016-5579-3

[30] T. Xie, Y. Wang, S. Fu et al. Predictors for intravenous immunoglobulin resistance and coronary artery lesions in Kawasaki disease[J]. Pediatr Rheumatol Online J, 2017, 15(1): 17.10.1186/s12969-0170149-1

[31] R. J. Bisoendial, S. M. Boekholdt, M. Vergeer et al. C-reactive protein is a mediator of cardiovascular disease[J]. Eur Heart J, 2010, 31(17): 2087-2091.10.1093/eurheartj/ehq238

[32] P. M. Ridker. Clinical application of C-reactive protein for cardiovascular disease detection and prevention[J]. Circulation, 2003, 107(3): 363-369.10.1161/01.cir.0000053730.47739.3c

[33] M. Süleymanoğlu, C. Burak, A. Gümüşdağ et al. Assessment of the relation between C-reactive protein to albumin ratio and the severity and complexity of peripheral arterial disease[J]. Vascular, 2020, 28(6): 731-738.10.1177/1708538120925952 
[34] M. Wei, M. Huang, S. Chen et al. A Multicenter Study of Intravenous Immunoglobulin Non-response in Kawasaki Disease[J]. Pediatr Cardiol, 2015, 36(6): 1166-1172.10.1007/s00246-015-1138-0

[35] P. P. Fu, Z. D. Du Y. S. Pan. Novel predictors of intravenous immunoglobulin resistance in Chinese children with Kawasaki disease[J]. Pediatr Infect Dis J, 2013, 32(8): e319-323.10.1097/INF.0b013e31828e887f

[36] K. Yasukawa, M. Terai, S. T. Shulman et al. Systemic production of vascular endothelial growth factor and fms-like tyrosine kinase-1 receptor in acute Kawasaki disease[J]. Circulation, 2002, 105(6): 766769.10.1161/hc0602.103396

[37] M. Terai, T. Honda, K. Yasukawa et al. Prognostic impact of vascular leakage in acute Kawasaki disease[J]. Circulation, 2003, 108(3): 325-330.10.1161/01.Cir.0000079166.93475.5f

[38] P. B. Soeters, R. R. Wolfe A. Shenkin. Hypoalbuminemia: Pathogenesis and Clinical Significance[J]. JPEN J Parenter Enteral Nutr, 2019, 43(2): 181-193.10.1002/jpen.1451

[39] T. Kobayashi, Y. Inoue, K. Takeuchi et al. Prediction of intravenous immunoglobulin unresponsiveness in patients with Kawasaki disease[J]. Circulation, 2006, 113(22): 26062612.10.1161/circulationaha.105.592865

[40] T. Yahata K. Hamaoka. Oxidative stress and Kawasaki disease: how is oxidative stress involved from the acute stage to the chronic stage?[J]. Rheumatology (Oxford), 2017, 56(1): 613.10.1093/rheumatology/kew044

[41] A. Franco, C. Shimizu, A. H. Tremoulet et al. Memory T-cells and characterization of peripheral T-cell clones in acute Kawasaki disease[J]. Autoimmunity, 2010, 43(4): $317-$ 324.10.3109/08916930903405891

[42] C. Shimizu, T. Oharaseki, K. Takahashi et al. The role of TGF- $\beta$ and myofibroblasts in the arteritis of Kawasaki disease[J]. Hum Pathol, 2013, 44(2): 189-198.10.1016/j.humpath.2012.05.004

[43] K. Sakata, K. Hamaoka, S. Ozawa et al. Matrix metalloproteinase-9 in vascular lesions and endothelial regulation in Kawasaki disease[J]. Circ J, 2010, 74(8): 1670-1675.10.1253/circj.cj-09-0980

[44] P. N. Jone, M. S. Anderson, M. J. Mulvahill et al. Infliximab Plus Intravenous Immunoglobulin (IVIG) Versus IVIG Alone as Initial Therapy in Children With Kawasaki Disease Presenting With Coronary Artery Lesions: Is Dual Therapy More Effective?[J]. Pediatr Infect Dis J, 2018, 37(10): 976980.10.1097/inf.0000000000001951

[45] H. Kariyazono, T. Ohno, V. Khajoee et al. Association of vascular endothelial growth factor (VEGF) and VEGF receptor gene polymorphisms with coronary artery lesions of Kawasaki disease[J]. Pediatr Res, 2004, 56(6): 953-959.10.1203/01.Pdr.0000145280.26284.B9

[46] Hy Lee Ms Song. Predictive factors of resistance to intravenous immunoglobulin and coronary artery lesions in Kawasaki disease[J]. Korean journal of pediatrics, 2016, 59(12): 477482.10.3345/kjp.2016.59.12.477 
Figures

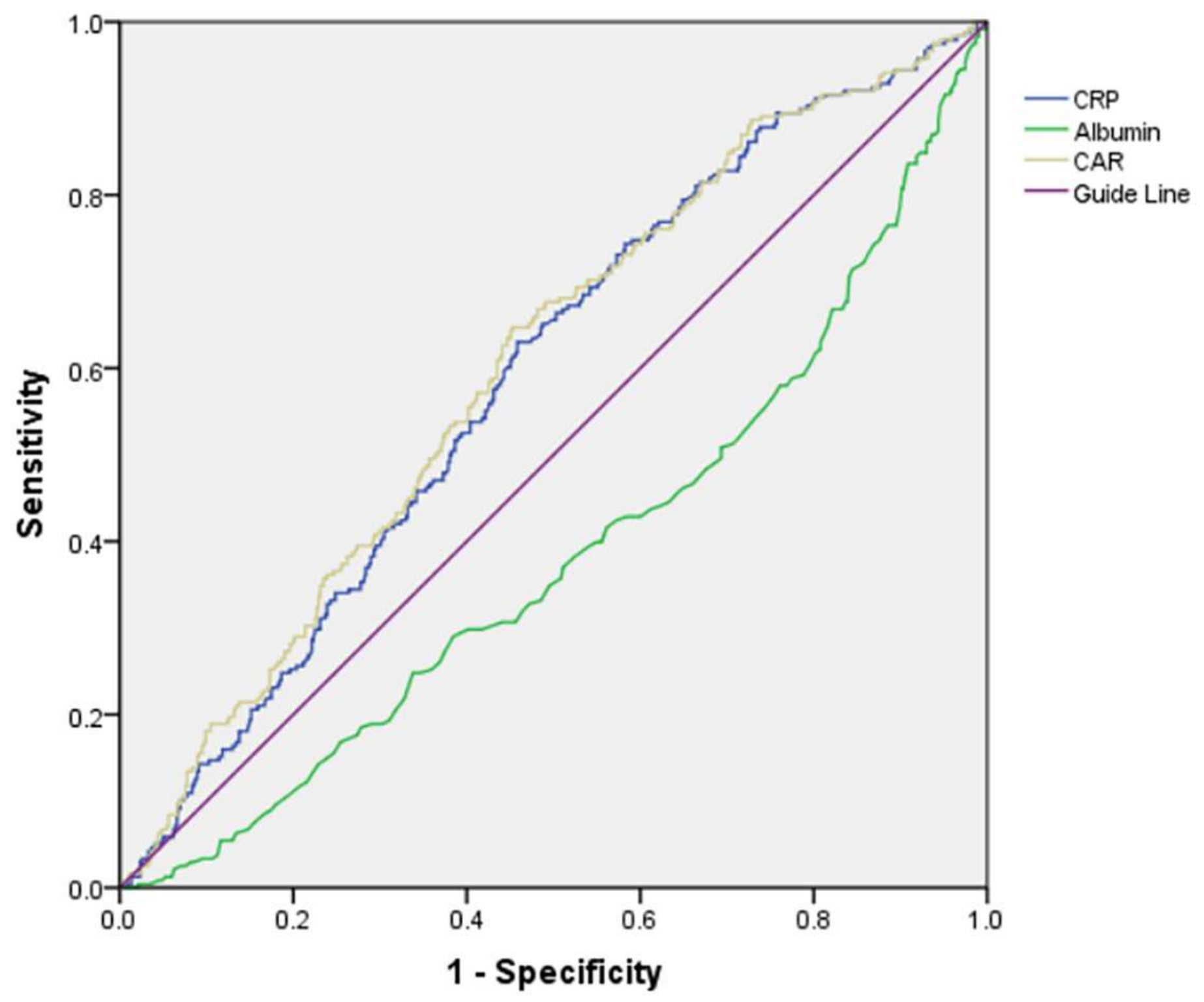

Figure 1

ROC curves of CAR and Coronary Artery Lesions 


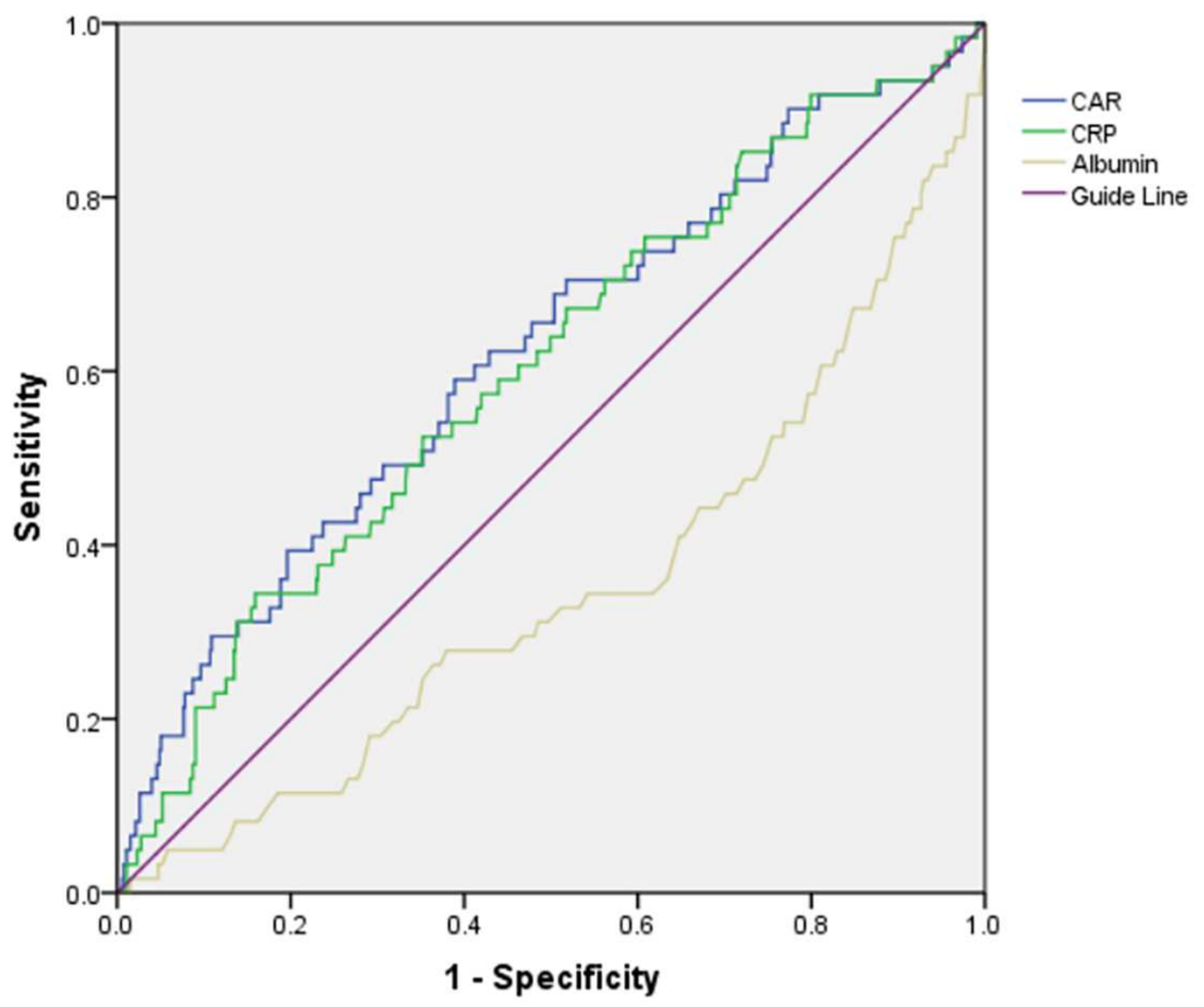

Figure 2

ROC curves of CAR and IVIG Resistance

\section{Supplementary Files}

This is a list of supplementary files associated with this preprint. Click to download.

- Table1.pdf

- Table2.pdf

- Table3.pdf

- Table4.pdf

- Table5.pdf 
- Table6.pdf 